\title{
Real Time Fault Tolerant Nonlinear Attitude Control System for Nanosatellite Applications
}

\author{
Junquan $\mathrm{Li}^{*}$ \\ Ryerson University, 350 Victoria St., Toronto, Ontario, M5B 2K3, Canada \\ Mark Post ${ }^{\dagger}$ and Regina Lee ${ }^{\ddagger}$ \\ York University, 4700 Keele Street Toronto, Ontario M3J 1P3, Canada
}

\begin{abstract}
This paper develops a fault tolerant attitude controller for next generation nanosatellites. The proposed fault tolerant attitude control algorithms in this study are based on first order and high order sliding mode control theory as well as fuzzy logic systems to achieve low cost and real time autonomy. A locally asymptotically stable adaptive fuzzy first order sliding mode controller is chosen as the best solution to the local attitude control tracking problem. This novel fault tolerant control is verified in the simulation results with reaction wheels' Coulomb friction, saturation, noise, dead-zone, bias fault and external disturbances. Simulation and testing results presented in the paper demonstrate that the attitude control system can provide successful pointing and tracking in the presence of system uncertainties for a specified class of reaction wheel failures.
\end{abstract}

\section{Nomenclature}

$J_{s} \quad$ Moment of inertia of satellite, $\mathrm{kgm}^{2}$

$m \quad$ Mass of satellite, $\mathrm{kg}$

$\omega \quad$ Angular velocity, $\mathrm{rad} / \mathrm{s}$

$J_{w} \quad$ Moment of inertia of $i$ th reaction wheel, $\mathrm{kgm}^{2}$

$\Omega \quad$ Angular velocity of $i$ th reaction wheel, $\mathrm{rad} / \mathrm{s}$

$\tau \quad$ Attitude control torque, $\mathrm{Nm}$

$\tau_{W} \quad$ Gravity gradient torque, $N m$

$d_{W} \quad$ External disturbances, $N m$

$\bar{q} \quad$ Quaternion of $i$ th satellite, vector component

$q_{4} \quad$ Quaternion of $i$ th satellite, scalar component

$e_{a} \quad$ Input voltage of reaction wheel, $V$

$\tau_{f} \quad$ Coulomb friction of reaction wheel, $\mathrm{Nm}$

$\mu \quad$ Fuzzy membership function

$\xi \quad$ Fuzzy basis function

\section{Introduction}

Nanosatellites (spacecraft with a total mass of 1-10 kg) are becoming important not only as a means to educate, but also as scientific instruments to make specific measurements of the earth cost effectively and with greater flexibility due to their availability. For most large satellites that use an active ACS, a pure simulation or hardware-in-the-loop system using a software environment like Matlab and Simlink is very useful. However, an autonomous ACS that can be used on nanosatellites should be tested in a real time simulation environment. A nonlinear discrete-time control algorithm needs to be investigated to manage the

*PhD Candidate, Department of Aerospace Engineering; junquan.li@ryerson.ca.

${ }^{\dagger} \mathrm{PhD}$ Candidate, Department of Earth and Space Engineering; markpost@yorku.ca.

‡Assistant Professor, Department of Earth and Space Engineering; reginal@yorku.ca. 
nonlinear satellite sensor and actuator dynamics, but there have been few actual implementations. With the development of smaller and cheaper micro-processors, autonomous nonlinear discrete control algorithms should be possible for an ACS.

In the reference, ${ }^{1}$ the reconfigurable fault-tolerant attitude control architecture and the associated Software and Hardware-in-the-Loop Test System for three-axis active control has been proposed at MIT. However, advanced control alogrithms have not been explored in detail. We aim to develop a next-generation non-linear attitude fault tolerant control system algorithm integrated with a novel spectrometer that will enable a cost-effective nanosatellite of $1-10 \mathrm{~kg}$ mass to perform detailed spectrometry measurements of the atmosphere for monitoring the emission and spreading of greenhouse gases such as carbon dioxide. To allow a small spectrometer to make these measurements autonomously from a nanosatellite, an adaptive nonlinear controller is required that is efficient and accurate enough to operate with the power, computation, and actuation limitations present on a nanosatellite while compensating for sensor and actuator nonlinearities to achieve the required arc-minute accuracy.

The satellite motion is governed by kinematic and dynamic equations. The mathematical models are highly nonlinear and linear feedback control techniques are not suitable for the system design because the accuracy and precision decreases due to the contribution of the nonlinear terms. A comparative study of various control methods such as $H_{2}$ and $H_{\infty}$ was applied for low Earth orbiting remote sensing satellites $(509 \mathrm{~kg}) .^{2}$ Most of the references ${ }^{3,4}$ for satellite tracking control did not consider hard nonlinearties such as Coulomb friction, saturations, noise, dead-zones and external disturbances. None of those references has given results for a small satellite with faults. Dynamics inversion and time-delay fault tolerant control methods have been developed for satellites with four reaction wheels and compared with PD controller. ${ }^{5}$ However, there is no analysis for the control accuracy performance with different initial conditions, different moments of inertia. Most of the fault tolerant control methods consider the system's ability to maintain its stability and performance with the fault. None of them presents control performance with a fault which is as good as the results without a fault. In the last several years, various structure control technologies have been used for the satellite tracking maneuver problem ${ }^{4,6}$ without faults. Fuzzy logic system is usually designed based on human expert knowledge in for of fuzzy rule. In most cases, we do not have enough expert knowledge in advance. The adaptive fuzzy methodology is used to build a proper fuzzy rule base. Fuzzy sliding mode controllers have been used for satellite attitude control systems ${ }^{7}$ for a fault free case. The simulation results show that the fuzzy sliding mode controller works better than an LQR controller and reduces fuel consumption. In this research, the first order sliding mode fault tolerant controller with adaptive fuzzy control is used and compared with two novel fault tolerant control laws based on high order sliding mode theory and fuzzy logic systems.

The main contributions of this study relative to other works are as follows:

1. A novel fault tolerant control method uses a sliding mode control scheme and fuzzy logic system for precise attitude tracking in the presence of unknown time-varying satellite actuator faults. This proposed control integrated fault tolerant control approach gives higher tracking precision and effectiveness than using other existing methods.

2. The novel fault tolerant control proposed in this paper is applied to a student-built air bearing satellite simulator controlled by reaction wheels. Tests are performed to determine the effectiveness of the control theories under nominal operating conditions and simulated actuator faults.

\section{Spacecraft Attitude Dynamics and Mathematical Model for Controller Design}

The spacecraft is modeled as a rigid body with reaction wheels that provide torques about three mutually perpendicular axes that defines a body-fixed frame $B$. The equations of motion are given by

$$
\begin{aligned}
\hat{J} \dot{\omega} & =-\omega^{\times}\left(J_{s} \omega+A_{i} J_{w} \Omega\right)+A_{i} \tau+d \\
\dot{q} & =\frac{1}{2}\left(\begin{array}{c}
q_{4} I_{3 \times 3}+\bar{q}^{\times} \\
-\bar{q}^{T}
\end{array}\right) \omega \equiv \frac{1}{2} A(q) \omega
\end{aligned}
$$


where $\omega=\left(\omega_{1}, \omega_{2}, \omega_{3}\right)^{T}$ is the angular velocity of the spacecraft with respect to an inertial frame $I$ and expressed in the body frame $B ; \Omega$ is the angular velocity of the reaction wheel. $J_{s} \in R^{3 \times 3}$ is the inertia matrix of the spacecraft; $\hat{J}=J_{s}-A_{i} J_{w} A_{i}^{T} ; \tau \in R^{3}$ is the torque control; $A_{i}$ is the $3 \times 4$ or $3 \times 3$ (depends on the layout and the number of the reaction wheels) matrix whose columns represent the influence of each reaction wheel on the angular acceleration of the satellite; $d \in R^{3}$ is the bounded external disturbance; $x^{\times} \in R^{3 \times 3}$ represents the cross product operator for a vector $x=\left(x_{1}, x_{2}, x_{3}\right)^{T}$ given as

$$
x^{\times}=\left(\begin{array}{ccc}
0 & -x_{3} & x_{2} \\
x_{3} & 0 & -x_{1} \\
-x_{2} & x_{1} & 0
\end{array}\right)
$$

and the unit quaternion $q=\left(\bar{q}^{T}, q_{4}\right)^{T}=\left(q_{1}, q_{2}, q_{3}, q_{4}\right)^{T}$ represents the attitude orientation of a rigid spacecraft in the body frame $B$ with respect to the inertial frame $I$, which is defined by

$$
\bar{q}=\left(q_{1}, q_{2}, q_{3}\right)^{T}=e \sin (\theta / 2), \quad q_{4}=\cos (\theta / 2)
$$

where $e$ is the Euler axis, and $\theta$ is the Euler angle. The unit quaternion $q$ satisfies the constraint

$$
q^{T} q=1
$$

The torques generated by the reaction wheels $\tau$ are given by

$$
\tau=J_{w}\left(\dot{\Omega}+A_{i}^{T} \dot{\omega}\right)
$$

Property 1: $A^{T} A=I_{3 \times 3}, \quad A^{T}(q) q=0$.

Property 2: The inertia matrix $J$ is a symmetric and positive definite matrix that satisfies the bounded condition given by

$$
J_{m}\|x\|^{2} \leq x^{T} J x \leq J_{M}\|x\|^{2}, \forall x \in R^{3}
$$

where $J_{m}$ and $J_{M}$ are positive constants, respectively.

To address the attitude tracking problem, the attitude tracking error $q_{e}=\left(\bar{q}_{e}^{T}, q_{4 e}\right)^{T}$ is defined as the relative orientation between the body frame $B$ and the desired frame $D$ with orientation $q_{d}=\left(\bar{q}_{d}^{T}, q_{4 d}\right)^{T}$, and it is computed by the quaternion multiplication rule as

$$
\begin{aligned}
\bar{q}_{e} & =q_{4 d} \bar{q}-q_{4} \bar{q}_{d}+\bar{q}^{\times} \bar{q}_{d} \\
q_{4 e} & =q_{4 d} q_{4}+\bar{q}_{d}^{T} \bar{q}
\end{aligned}
$$

where $q_{d} \in R^{4}$ and $q_{e} \in R^{4}$ satisfy the constraints $q_{d}^{T} q_{d}=1$ and $q_{e}^{T} q_{e}=1$, respectively. The corresponding rotation matrix is given by

$$
C\left(q_{e}\right)=\left(q_{4 e}^{2}-\bar{q}_{e}^{T} \bar{q}_{e}\right) I_{3 \times 3}+2 \bar{q}_{e} \bar{q}_{e}^{T}-2 q_{4 e} \bar{q}_{e}^{\times}
$$

Note that $\|C\|=1$ and $\dot{C}=-\omega_{e}^{\times} C$, where $\omega_{e}=\omega-C \omega_{d}$ is the relative angular velocity of $B$ with respect to $D$, and $\omega_{d} \in R^{3}$ is the desired angular velocity.

The control objective is to design the controller $\tau$ such that all signals involved in the resulting closed-loop system are uniformly ultimately bounded.

$$
\ddot{\bar{q}}=2\left(T^{-1}\right)^{T}\left[\hat{J}^{-1}\left(-\omega \times\left(J_{s} \omega+A_{i} J_{w} \Omega\right)+A_{i} \tau+d\right]-4\left(T^{-1}\right)^{T}\left(\dot{T}^{-1}\right) \dot{\bar{q}}\right.
$$

where $T=\left[q_{4} I+(\bar{q})^{\times}\right]^{-1}$.

The dynamics error equation with actuator fault can be written as:

$$
\ddot{\bar{q}}_{e}=f\left(\bar{q}_{e}, \dot{\bar{q}}_{e}\right)+(\hat{\tau}+\Delta \tau)+\nu
$$

A dynamic model of the satellite's attitude tracking with multiplicative reaction wheel faults (e.g. reduced control torque) is represented by a diagonal matrix $E$.

$$
\begin{aligned}
f_{i}\left(\bar{q}_{e}, \dot{\bar{q}}_{e}\right) & =2\left(T^{-1}\right)^{T}\left[J_{i}{ }^{-1}\left(-\omega_{\times}\left(J_{s} \omega+A_{i} J_{w} \Omega\right)\right]-4\left(T^{-1}\right)^{T}\left(\dot{T}^{-1}\right) \dot{q}_{i e}\right. \\
\hat{\tau} & =E\left[2\left(T^{-1}\right)^{T} J_{i}{ }^{-1} A_{i} \tau\right] \\
\nu & =2\left(T^{-1}\right)^{T} J_{i}{ }^{-1} d
\end{aligned}
$$




\section{Fault Tolerant Control Design}

The linear sliding mode (LSM), the initial terminal sliding mode (TSM), and the nonsingular TSM (NTSM) can be described by the following first-order nonlinear differential equations:

$$
\begin{aligned}
\mathrm{LSM}: & s=\dot{x}+k x \\
\mathrm{TSM}: & s=\dot{x}+k x^{q / p} \\
\mathrm{NTSM}: & s=x+k^{-p / q} \dot{x}^{p / q}
\end{aligned}
$$

respectively, where $x \in R ; k$ is a positive constant, and the positive odd integers $p, q$ are chosen such that $1<p / q<2$.

The nonsingular fast terminal sliding mode can be defined by the following nonlinear differential equation:

$$
s=\bar{q}_{e}+\sigma_{1} \operatorname{sign}\left(\bar{q}_{e}\right)+\sigma_{2} \dot{\bar{q}}_{e}^{\frac{p}{q}}
$$

We rewrite Eq. (19) as

$$
s=\Lambda+\beta
$$

where $\Lambda=\bar{q}_{e}+\sigma_{1} \operatorname{sign}\left(\bar{q}_{e}\right)$ and $\beta=\sigma_{2} \dot{q}_{e} \frac{p}{q}$.

The time derivative of the sliding mode Eq. (20) is given by

$$
\dot{s}=\dot{\Lambda}+\dot{\beta}
$$

The continuous nonlinear function $\Delta \tau$ of the satellite can be approximated by fuzzy logic system.

$$
\Delta \hat{\tau}=\theta^{T} \xi(x)
$$

where $\xi_{F}$ is the vector of the fuzzy basis function and is assumed to be fixed, while the parameters $\theta_{F}^{T}$ are the variable which will be designed by adaptive laws. It is assumed that there exists an optimal fuzzy logic system to learn the nonlinear terms $\Delta \tau$ such that

$$
\Delta \tau-\Delta \hat{\tau}^{*}=\varepsilon
$$

where $\varepsilon$ is the approximation error and is bounded. Approximation errors can be reduced by increasing the number of fuzzy rules.

In order to decrease the number of fuzzy rules needed, we use the nonsingular fast terminal sliding surface $s$ instead of the state $x$ for the adaptive parameters. Then, the adaptive parameters $\theta$ are updated by the following adaptive laws: Then, the adaptive parameters $\theta$ are updated by the following adaptive laws:

$$
\begin{gathered}
\dot{\theta}=\alpha \sigma_{2} \rho \dot{\bar{q}}_{e}^{\rho-1} s \xi \\
\xi=\frac{\prod_{i=1}^{N} \mu_{A i^{l}(s)}}{\sum_{l=1}^{P} \prod_{i=1}^{N} \mu_{A i^{l}(s)}}
\end{gathered}
$$

where $\alpha$ is a positive constant and $\rho=p / q$. In order to guarantee that the estimated Fuzzy Logic System parameters remain within some known bounded sets, a smooth projection is considered in this chapter. Let $\theta$ be the estimation of the optimal weight matrix $\theta^{*}$, and define a smooth projection of $\theta$ as:

$$
\pi(\theta)=\theta_{\pi}=\pi_{j k}\left(\theta_{j k}\right), j=1,2, \ldots, m, k=1,2, \ldots, N_{1}
$$

Each projection operator $\pi_{j k}$ is a real-value smooth nondecreasing function defined by

$$
\pi_{j k}\left(\theta_{j k}\right)=\theta_{j k}, \forall \theta_{j k} \in\left[\theta_{\min }, \theta_{\max }\right] \pi_{j k}\left(\theta_{j k}\right) \in\left[\theta_{\min }-\varepsilon_{M i}, \theta_{\max }+\varepsilon_{M i}\right]
$$

Define $\tilde{\theta}=\theta^{*}-\theta, \tilde{\theta}_{\pi}=\theta^{*}-\theta_{\pi}$, and

$$
V_{\theta}=\frac{1}{\delta} \sum_{j=1}^{m} \sum_{k=1}^{N_{1}} \int_{0}^{\tilde{\theta}}\left(\theta^{*}{ }_{j k}-\pi_{j k}\left(\theta^{*}{ }_{j k}-\omega_{j k}\right) d \omega_{j k}, i=1,2, \ldots, n\right.
$$


where, $V_{\theta}$ is positive definite with respect to $\tilde{\theta}_{j k}$ for $\theta_{j k}^{*} \in\left[\theta_{\min , \theta_{\max }}\right]$. Furthermore,

$$
\dot{V}_{\theta}=-\frac{1}{\delta} \sum_{j=1}^{m} \sum_{k=1}^{N_{1}} \tilde{\theta}_{\pi, j k} \dot{\theta}_{j k}^{*}, i=1,2, \ldots, n
$$

The proposed attitude fault tolerant controller (AFSMC: fuzzy sliding mode control) design for a satellite is now given by

$$
\hat{\tau}=-k_{1} s-k_{2} s^{\rho}-\frac{q}{p} s^{2-p / q}-\theta^{T} \xi-\varphi
$$

$\varphi$ defined by ${ }^{8}$

$$
\varphi \equiv \kappa \tanh \left(\frac{3 K_{u} \kappa s}{\epsilon}\right)
$$

\section{Stability Analysis}

Theorem: In the system Eq. (12), the nonsingular fast fuzzy terminal sliding control laws defined by Eqs. (30-31), with adaptive control laws given by Eq. (24) guarantee that all the signals of the closed loop system are bounded during actuator faults.

Proof of Theorem: The dynamics equations for the quaternion errors of the satellite can be written as:

$$
\begin{gathered}
\bar{q}_{e}=e_{1} \quad \dot{\bar{q}}_{e}=e_{2} \\
\dot{e}_{1}=e_{2} \quad \dot{e}_{2}=f\left(\bar{q}_{e}, \dot{\bar{q}}_{e}\right)+\hat{\tau}+\Delta \tau+\nu
\end{gathered}
$$

Using Eq. (21), we obtained

$$
\dot{s}=\dot{e}_{1}+\sigma_{2} \rho \dot{e}_{1}^{\rho-1}(f+\hat{\tau}+\Delta \tau+\nu)
$$

Using the approximation property of Fuzzy Logic Systems, the unknown function $f$ can be approximated over the compact set $\Omega_{d}$ by Eq. (22). Substituting the control law Eqs. (30-31) into the error dynamics Eq. (12) yields the following dynamic equation for sliding surface $s$.

$$
\begin{aligned}
& \dot{s}=\dot{e}_{1}+\sigma_{2} \rho \dot{e}_{1}^{\rho-1}\left(F-F_{d}+F_{d}-\theta^{T} \xi-K_{1} s-K_{2} s^{\rho}\right. \\
&\left.-\frac{1}{\rho \sigma_{2}} \dot{e}_{1}^{2-\rho}-\varphi+\nu+\Delta \tau\right) \\
&= \sigma_{2} \rho \dot{e}_{1}^{\rho-1}\left(\varsigma+\tilde{\theta}^{T} \xi-K_{1} s-K_{2} s^{\rho}-\varphi+\nu+\varepsilon+\Delta \tau\right)
\end{aligned}
$$

where $\varsigma=F-F_{d}$.

Consider the following Lyapunov function candidate

$$
V=\frac{1}{2} s^{T} s+V_{\theta_{i}}
$$

Thus, the time derivative of $V$ is obtained as

$$
\dot{V}=s^{T} \dot{s}+\dot{V}_{\theta_{i}}
$$

Due to the fact that

$$
s^{T} \sigma_{2} \rho \dot{e}_{1}^{\rho-1} \tilde{\theta}^{T} \xi-\frac{1}{\delta} \sum_{j=1}^{m} \sum_{k=1}^{N_{1}} \tilde{\theta}_{F_{\pi, j k}} \dot{\theta}_{j k}^{*}=0
$$

Substituting Eqs. (29) and (35) into Eq. (37), we obtain:

$$
\begin{gathered}
\dot{V}=\sigma_{2} \rho \dot{e}_{1}^{\rho-1}\left(s^{T} \varsigma+s^{T} \tilde{\theta}^{T} \xi-s^{T} K_{1} s-s^{T} K_{2} s^{\rho}-s^{T} \varphi\right. \\
\left.+s^{T}(\nu+\varepsilon+\Delta \tau)-\frac{1}{\delta} \sum_{j=1}^{m} \sum_{k=1}^{N_{1}} \tilde{\theta}_{F_{\pi, j k}} \dot{\theta}_{j k}^{*}\right) \\
=\sigma_{2} \rho \dot{e}_{1}^{\rho-1}\left(s^{T} \varsigma-s^{T} K_{1} s-s^{T} K_{2} s^{\rho}-s^{T} \varphi\right. \\
\left.+s^{T}(\nu+\varepsilon+\Delta \tau)\right)
\end{gathered}
$$


Using the inequality in the reference, ${ }^{9}$ it is easy to obtain that

$$
-s^{T} \varphi+s^{T}(\nu+\varepsilon+\Delta \tau) \leq n \epsilon
$$

Using the well-known inequality:

$$
\left(\sqrt{c_{5}} s-\frac{\varsigma}{2 \sqrt{c_{5}}}\right)^{T}\left(\sqrt{c_{5}} s-\frac{\varsigma}{2 \sqrt{c_{5}}}\right) \geq 0
$$

The following inequality can be rewritten as

$$
s^{T} \varsigma \leq c_{5} s^{T} s+\frac{\varsigma^{2} M}{4 c_{5}}
$$

where $c_{5}$ is a positive constant satisfying $c_{5}<\lambda_{\min }\left(K_{1}\right)$ with $\lambda_{\min }\left(K_{1}\right)$ being the minimum eigenvalue of the matrix $K_{1}$. Eq. (39) becomes

$$
\begin{aligned}
\dot{V} \leq \sigma_{2} \rho \dot{e}_{1}^{\rho-1}( & \left.-\left(\lambda_{\min }\left(K_{1}\right)-c_{5}\right) s^{T} s+c_{4}-s^{T} K_{2} s^{\rho}\right) \\
\leq & -\sigma_{2} \rho \dot{e}_{1}^{\rho-1} M_{2 i}|s|^{2} \leq 0
\end{aligned}
$$

where $c_{4}=n \epsilon+\frac{\varsigma^{2} M}{4 c_{5}}$ is a positive constant.

Inequality Eq. (43) implies that the sliding surfaces are bounded. The state errors $\bar{q}_{e}$ and $\dot{q}_{e}$ are bounded. All signals in the closed-loop system are uniformly ultimately bounded. Thus, we have demonstrated the stability of the overall closed-loop system and have given the stability proof.

\section{Simulation Results}

The simulation of the three proposed fault tolerant controller has been performed. The computing environment for this simulation is: Matlab R2009b (64bit), running on processor (core speed of 1596[MHz]) and 1 GB of RAM memory (bus speed of $199.6[\mathrm{MHz}]$ ). The simulation results were presented in the reference $^{10}$ to illustrate the effectiveness of the control strategies. The total disturbances acting on the satllite include gravity gradient torque $\tau_{M}$ and external disturbances $d_{M}$. The external disturbances are chosen as $(0.01)[\sin (0.8 t) ; \cos (0.5 t) ; \cos (0.3 t)] \mathrm{Nm}$. The dead zone and friction force on the reaction wheels are $1 \mathrm{~V}$ and $0.002 \mathrm{Nm}$ respectively. The maximum value for control input noise is $0.005 \mathrm{Nm}$. The other two modified existing attitude fault tolerant controllers using second order sliding mode control (2nd SMC) and third order sliding mode control $(3 \mathrm{rd} \mathrm{SMC})^{6}$ for a satellite are now given by

$$
\begin{gathered}
\hat{\tau}=-k \frac{\dot{s}+|s|^{1 / 2} \operatorname{sign}(s)}{\dot{s}+|s|^{1 / 2}}-\varphi-\theta^{T} \xi \\
\hat{\tau}=-\hat{k} \frac{\ddot{s}+2\left(|\dot{s}|+|s|^{2 / 3}\right)^{-1 / 2}\left(\dot{s}+|s|^{2 / 3} \text { signs }\right)}{|\ddot{s}|+2\left(|\dot{s}|+|s|^{2 / 3}\right)^{1 / 2}}-\varphi-\theta^{T} \xi
\end{gathered}
$$

It is difficult to find $\dot{s}$ and $\ddot{s}$. For Eq. 44, a first-order real-time differentiator ${ }^{11} Q_{0}$ and $Q_{1}$ is used in to estimate $s$ and $\dot{s}$.

$$
\begin{aligned}
\dot{Q}_{0} & =-\lambda_{1}\left|Q_{0}-s\right|^{1 / 2} \operatorname{sign}\left(Q_{0}-s\right)+Q_{1} \\
\dot{Q}_{1} & =-\lambda_{2} \operatorname{sign}\left(Q_{0}-s\right) \\
\varphi & \equiv \kappa \quad \tanh \left(\frac{3 K_{u} \kappa Q_{0}}{\epsilon}\right)
\end{aligned}
$$

For Eq. 45, a second-order real-time differentiator ${ }^{11} Q_{0}, Q_{1}$ and $Q_{2}$ is used in to estimate $s, \dot{s}$ and $\ddot{s}$.

$$
\begin{aligned}
\dot{Q}_{0} & =Z_{0} \\
Z_{0} & =-\hat{\lambda}_{1}\left|Q_{0}-s\right|^{2 / 3} \operatorname{sign}\left(Q_{0}-s\right)+Q_{1} \\
\dot{Q}_{1} & =Z_{1} \\
Z_{1} & =-\hat{\lambda}_{2}\left|Q_{1}-Z_{0}\right|^{1 / 2} \operatorname{sign}\left(Q_{1}-Z_{0}\right)+Q_{2} \\
\dot{Q}_{2} & =-\hat{\lambda}_{3} \operatorname{sign}\left(Q_{2}-Z_{1}\right) \\
\varphi & \equiv \kappa \tanh \left(\frac{3 K_{u} \kappa Q_{0}}{\epsilon}\right)
\end{aligned}
$$


Table 1. Parameters of Satellite

\begin{tabular}{l|c}
\hline Parameters & Values \\
\hline mass $(\mathrm{kg})$ & 2 \\
$\mu_{e}\left(\mathrm{~km}^{3} \mathrm{~s}^{-2}\right)$ & 398600 \\
$r_{L}(\mathrm{~km})$ & 6878 \\
Size $\left(\mathrm{m}^{3}\right)$ & $0.1 \times 0.1 \times 0.1$ \\
Desired Attitude Trajectory & $q_{d}=[0,0.005 \cos (0.2 t), 0.005 \sin (0.2 t)]^{T}$ \\
Initial Relative Angular Velocity & $\omega(0)=(0,0,0)^{T}(\mathrm{rad} / \mathrm{s})$ \\
Initial Quaternion (Satellite) & $q_{(10)}=(0.6,-0.5,0.6)^{T}$ \\
Moment of Inertia of Satellite $\left(\mathrm{kgm}^{2}\right)$ & $J=\operatorname{diag}(0.015,0.017,0.020)$ \\
Moment of Inertia of Wheels $\left(\mathrm{kgm}^{2}\right)$ & $J_{w}=10^{-5} \operatorname{diag}(1,1,1,1)$ \\
Maximum Voltage of Wheels $(V)$ & $e_{a}=4.5$ \\
\hline
\end{tabular}

Table 2. AFSMC Controller Parameters

\begin{tabular}{l|c}
\hline Parameters & Values \\
\hline$p, q$ & 11,9 \\
$\kappa, \epsilon$ & $0.1,0.01$ \\
$\sigma_{i 1}, \sigma_{i 2}$ & $0.00001,100$ \\
$k_{1}, k_{2}$ & $\operatorname{diag}(0.004,0.004,0.004), \operatorname{diag}(0.0025,0.0025,0.0025)$ \\
$\theta_{0}, \theta_{\min }, \theta_{\max }, \alpha$ & $0,-200,200,2$ \\
\hline
\end{tabular}

Table 3. 2nd SMC Controller Parameters

\begin{tabular}{l|c}
\hline Parameters & Values \\
\hline$p, q, k$ & $11,9,0.0009$ \\
$\kappa, \epsilon$ & $0.1,0.001$ \\
$\lambda_{1}, \lambda_{2}$ & 120,128 \\
$\theta_{0}, \theta_{\min }, \theta_{\max }, \alpha$ & $0,-200,200,9$ \\
\hline
\end{tabular}

Table 4. 3rd SMC Controller Parameters

\begin{tabular}{l|c}
\hline Parameters & Values \\
\hline$p, q, \hat{k}$ & $11,9,0.009$ \\
$\kappa, \epsilon$ & $0.1,0.001$ \\
$\hat{\lambda}_{1}, \hat{\lambda}_{2}, \hat{\lambda}_{3}$ & $20,26,81$ \\
$\theta_{0}, \theta_{\min }, \theta_{\max }, \alpha$ & $0,-200,200,9$ \\
\hline
\end{tabular}

Seven Gaussian membership functions are defined for each variable $\tilde{A}_{i}, i=1,2, \cdots, 7$ as:

$$
\begin{aligned}
& \mu_{\tilde{A}_{1}}\left(s_{i}\right)=\frac{1}{1+\exp \left(5\left(s_{i}+0.9 \times a\right)\right)} \\
& \mu_{\tilde{A}_{2}}\left(s_{i}\right)=\exp \left(-\left(\frac{s_{i}+0.6 \times a}{b}\right)^{2}\right), \quad \mu_{\tilde{A}_{3}}\left(s_{i}\right)=\exp \left(-\left(\frac{s_{i}+0.3 \times a}{b}\right)^{2}\right), \\
& \mu_{\tilde{A}_{4}}\left(s_{i}\right)=\exp \left(-\left(\frac{s_{i}}{b}\right)^{2}\right), \quad \mu_{\tilde{A}_{5}}\left(s_{i}\right)=\exp \left(-\left(\frac{s_{i}-0.3 \times a}{b}\right)^{2}\right) \\
& \mu_{\tilde{A}_{6}}\left(s_{i}\right)=\exp \left(-\left(\frac{s_{i}-0.6 \times a}{b}\right)^{2}\right)
\end{aligned}
$$




$$
\mu_{\tilde{A}_{7}}\left(s_{i}\right)=\frac{1}{1+\exp \left(5\left(s_{i}-0.9 \times a\right)\right)}
$$

Tracking error performance using AFSMC, 2ndSMC and 3rdSMC controllers with faults are shown in Table 5. The best controller for the fault free case is the 3rd SMC because the tracking error and control gain are the smallest. For the fault case, the 3rd SMC with a FLS is not the most robust controller, as the tracking error is the largest due to the fault. The 2nd SMC with a FLS controller is more robust, and the tracking error is smaller than that of the 3rd SMC due to the fault. However, the AFSMC controller is the best controller for the fault case.

Table 5. Summary of Tracking Error Performance for AFSMC, 2nd SMC and 3rd SMC Controller with Multiple Faults and Reaction Wheel Friction $(t=500 s)$

\begin{tabular}{|c|l|c|c|}
\hline Controller & Roll & Pitch & Yaw \\
\hline \hline AFSMC & $0.041^{\circ}$ & $0.045^{\circ}$ & $0.074^{\circ}$ \\
\hline 2nd SMC & $0.091^{\circ}$ & $0.105^{\circ}$ & $0.109^{\circ}$ \\
\hline 3rd SMC & $0.29^{\circ}$ & $0.21^{\circ}$ & $0.68^{\circ}$ \\
\hline
\end{tabular}

In Table 6, the quaternion norm and angular velocity norm of the proposed controllers (for fault free case) are compared with the existing reference. ${ }^{3}$ In most cases, the tracking performance shown in the references is degraded heavily by faults. The tracking performace is usually not so good due to the fault. However, the quaternion norm and angular velocity norm of the proposed controller AFSMC are 10.4e -5 and 5.3e -4 . These results are almost as good as the results in the reference ${ }^{3}$ which are $9.6 e-5$ and $5.75 e-4$.

Table 6. Summary of Tracking Error Performance for AFSMC, 2nd SMC and 3rd SMC Controller with the Existing Reference $^{3}(t=500 s)$

\begin{tabular}{|c|c|c|}
\hline Controller & Quaternion Norm & Angular Velocity Norm \\
\hline \hline AFSMC & $10.5 e-5$ & $5.3 e-4$ \\
\hline 2nd SMC & $9 e-4$ & $3 e-3$ \\
\hline 3rd SMC & $6 e-3$ & $2 e-3$ \\
\hline Controller of Kim 2003 & $9.64 e-5$ & $5.75 e-4$ \\
\hline Controller of Wie 1985 & 0.1039 & 0.1170 \\
\hline Controller of Joshi 1995 & 0.1957 & 0.1402 \\
\hline Controller of Krstic 1999 & 0.2368 & 0.1327 \\
\hline
\end{tabular}

The simulation results of AFSMC, 2nd SMC, and 3rd SMC are shown in Figs. 1, 2, 3, 4 and 5. 

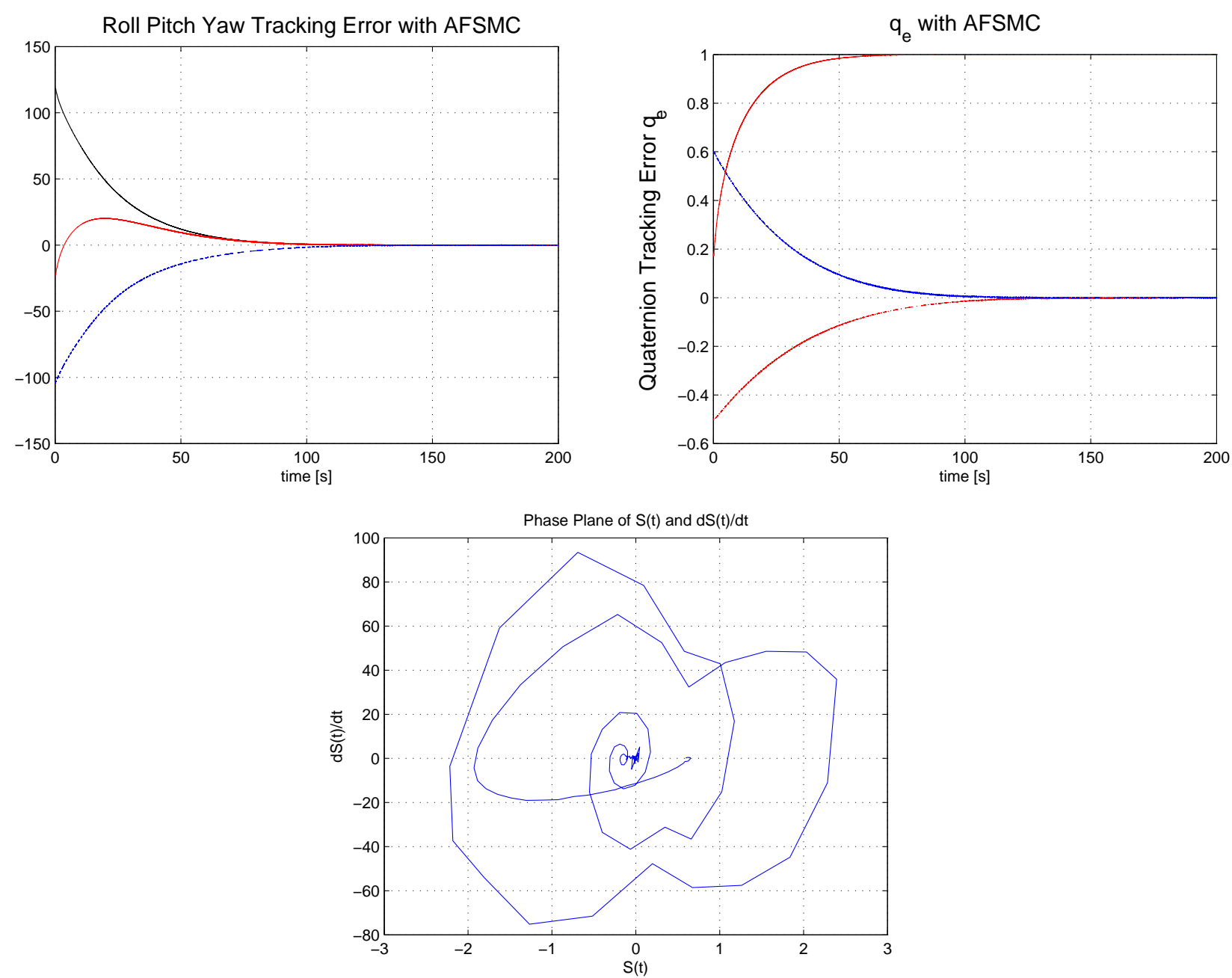

Figure 1. Angular Tracking Error, Quaternion Tracking Error, Angular Velocity Tracking Error without Fault Case using AFSMC Controller
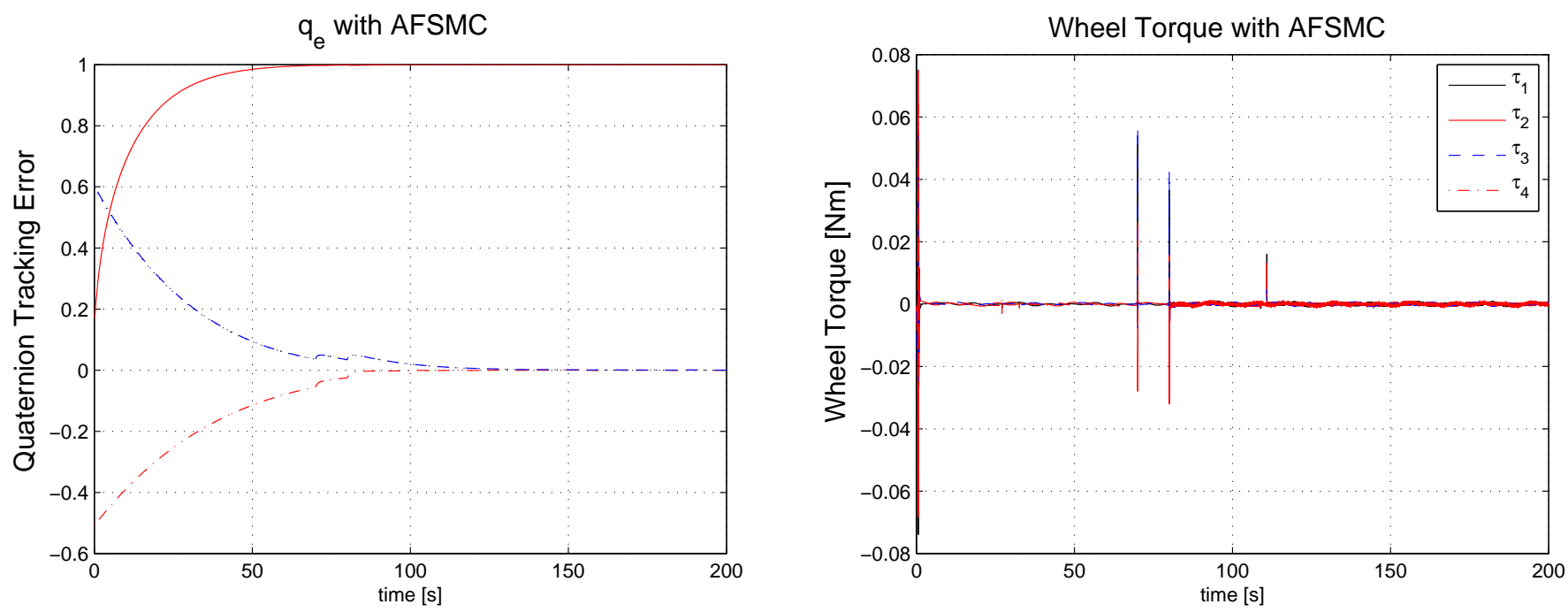

Figure 2. Quaternion Tracking Error and Wheel Torques Results using AFSMC controller: t=70s, Actuator Fault; $\mathrm{t}=80 \mathrm{~s}$, Actuator noise 

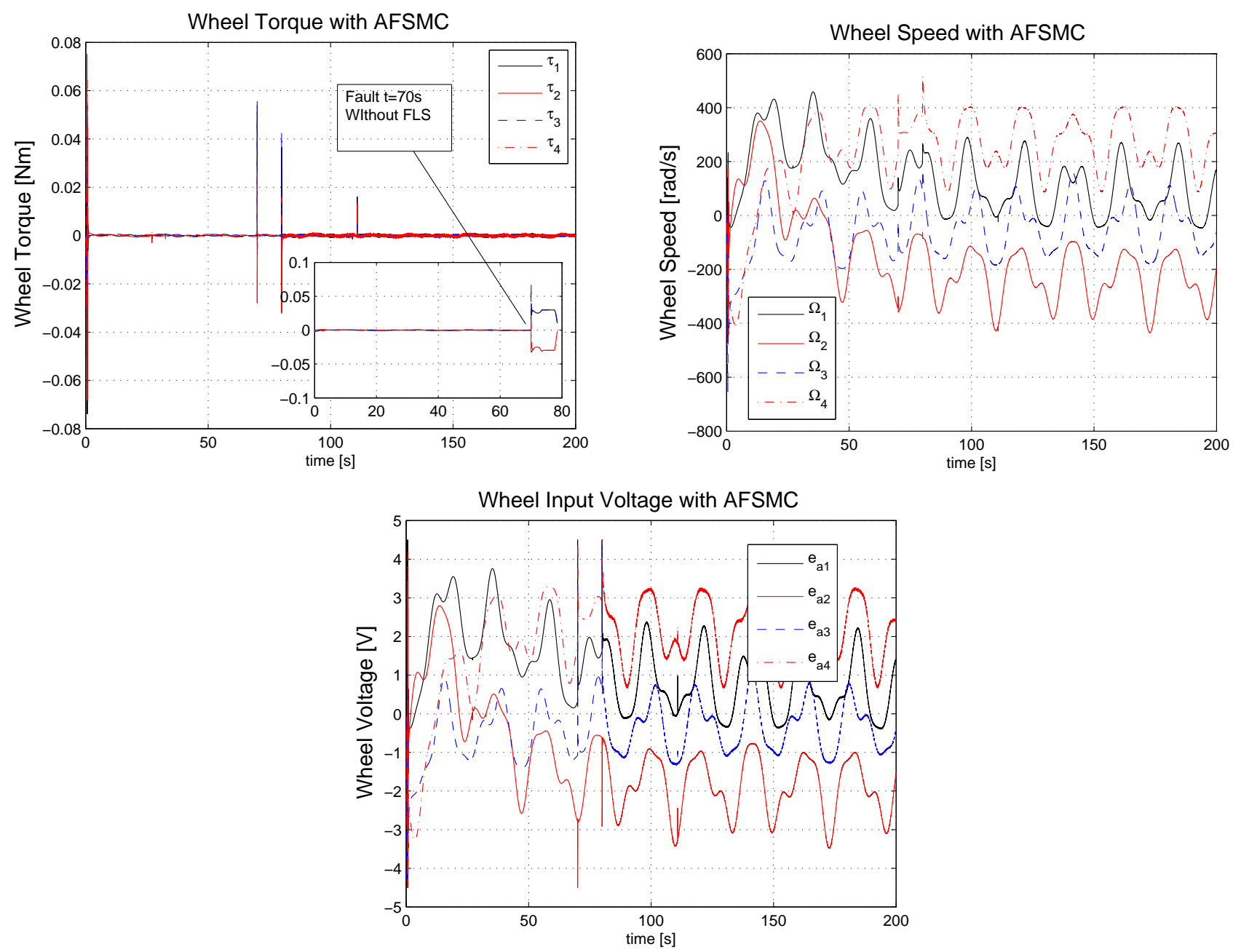

Figure 3. Wheel Voltage, Wheel Speed and Wheel Torques Fault Tolerance Results using AFSMC Controller: $t=70$ s, Actuator Fault; $t=80$ s, Actuator noise 

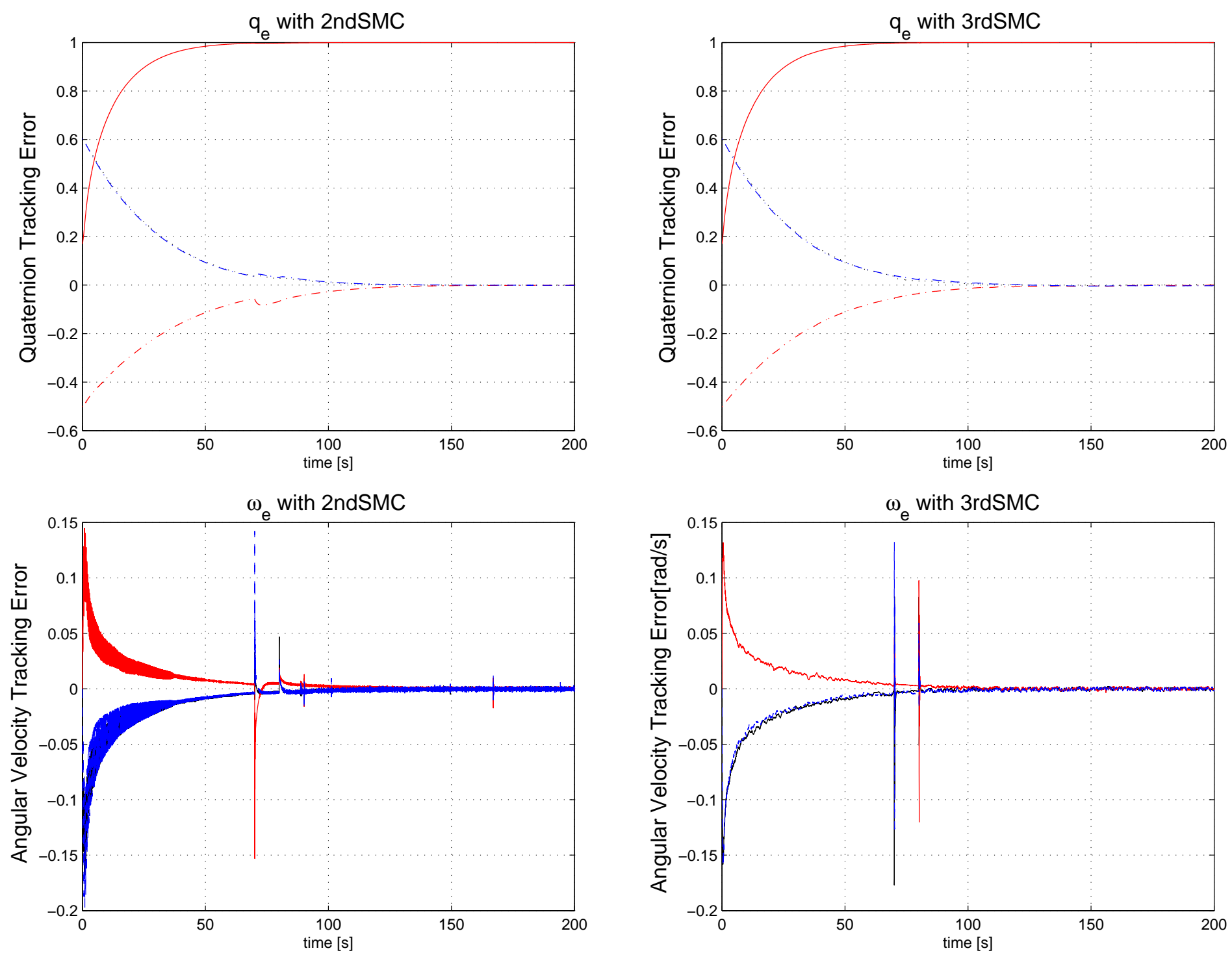

Figure 4. Quaternion Tracking Error, Angular Velocity Tracking Error Fault Tolerance Results using 2nd SMC Controller and 3rd SMC Controller : $\mathrm{t}=70 \mathrm{~s}$, Actuator Fault; $\mathrm{t}=80 \mathrm{~s}$, Actuator noise 

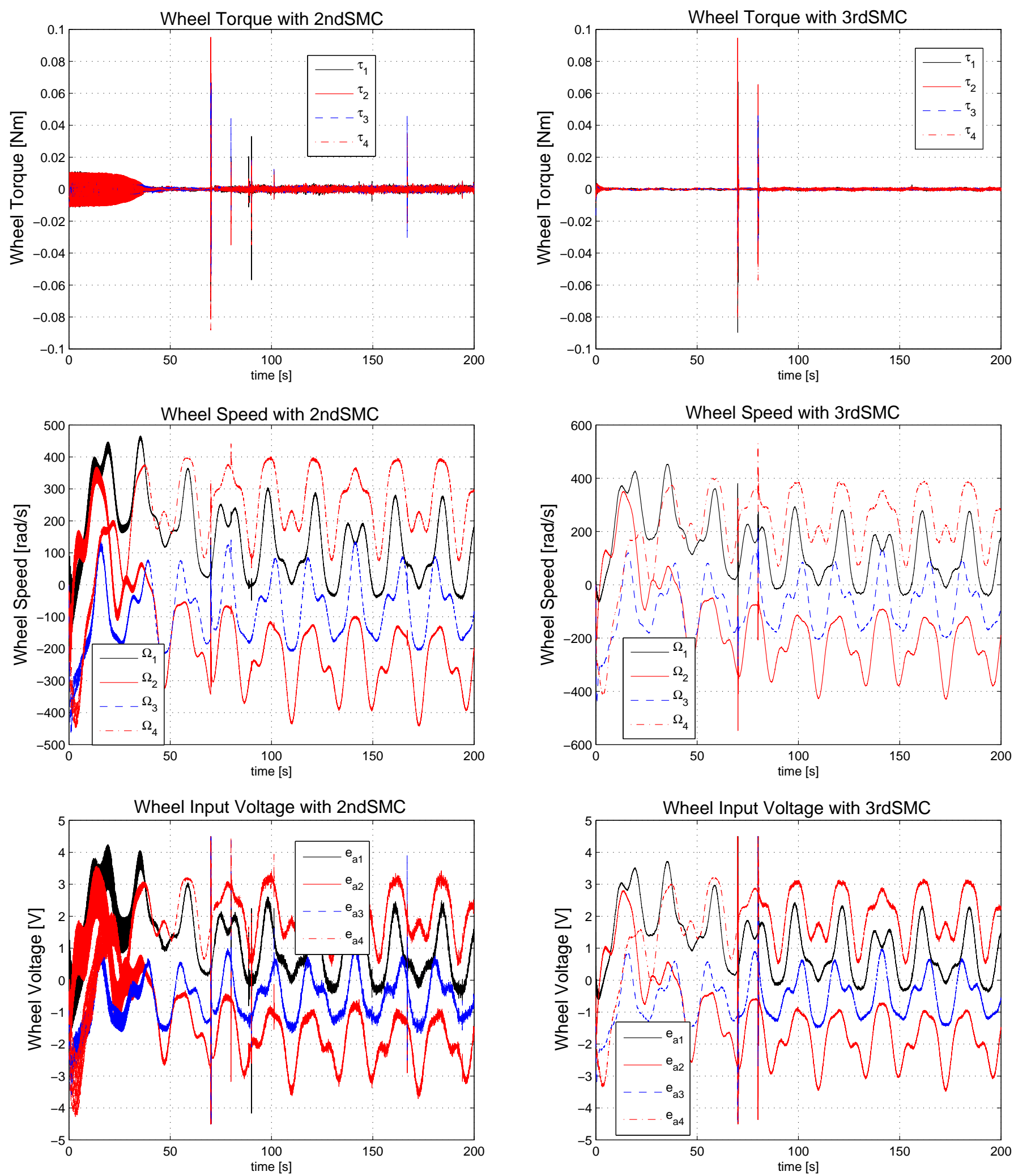

Figure 5. Wheel Voltage, Wheel Speed and Wheel Torques Fault Tolerance Results using 2nd SMC Controller and 3rd SMC Controller: $t=70 \mathrm{~s}$, Actuator Fault; $t=80 \mathrm{~s}$, Actuator noise 


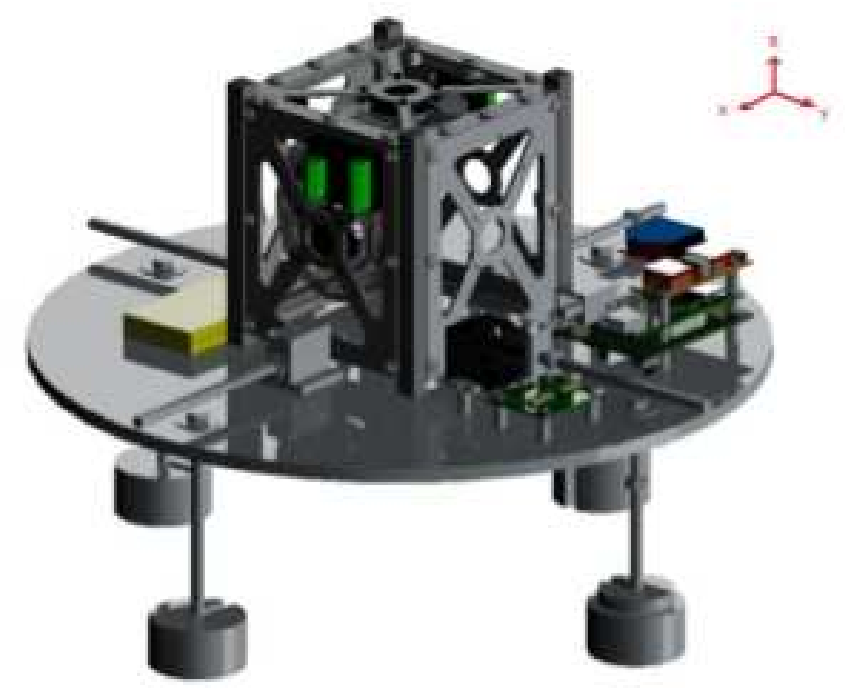

Figure 6. Spherical Air Bearing System [Space Engineering Lab in York University]

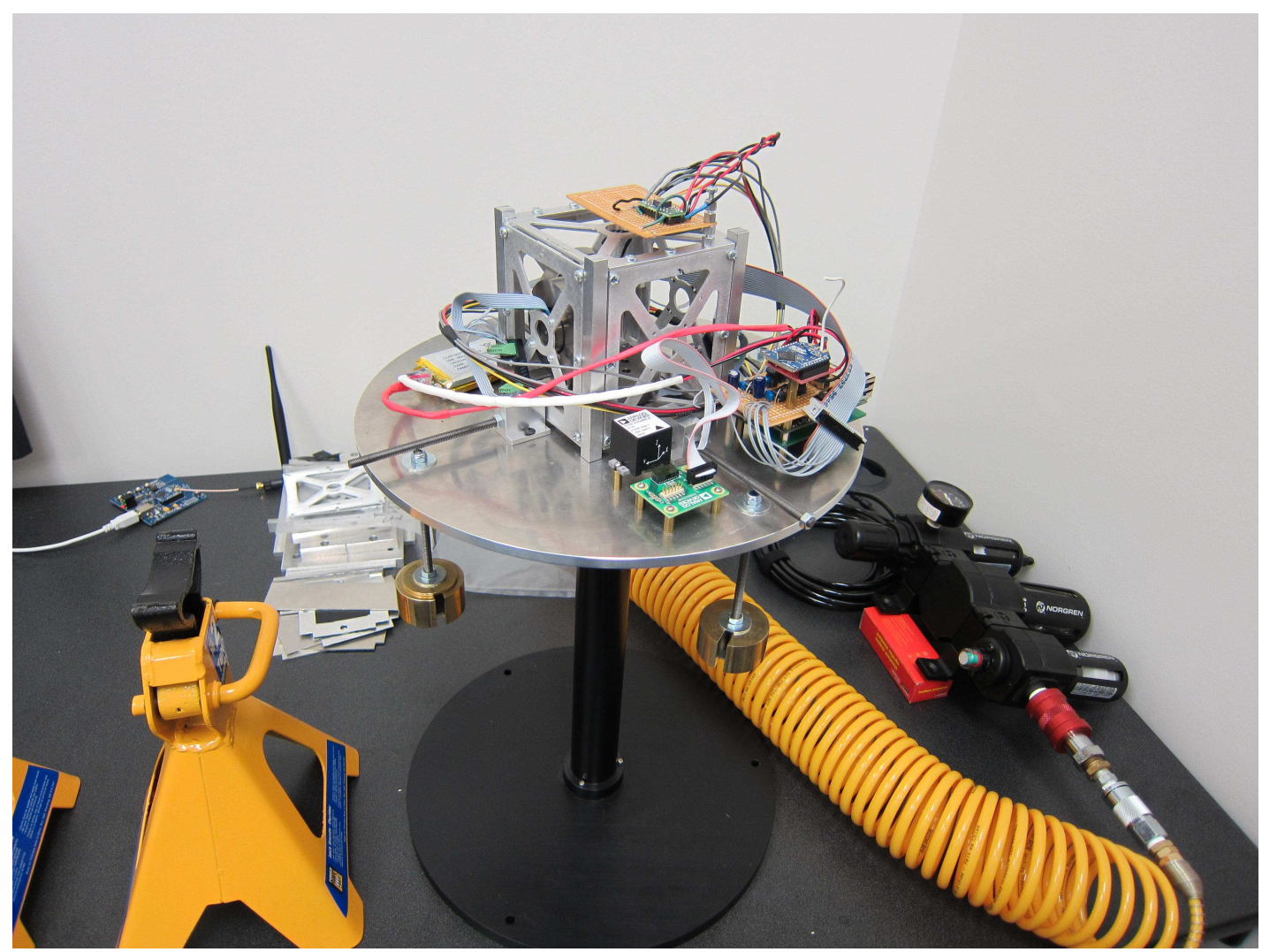

Figure 7. Spherical Air Bearing Setup with Electronics System and Control System [Space Engineering Lab in York University] 


\section{Nanosatellite Air Bearing Testing in York University}

The current research is limited in terms of actual implementation. Accurately simulating the environment of outer space on Earth for testing of space hardware is a very difficult challenge. This complicates the process of hardware validation, since hardware performance must be evaluated during each test while performing the function appropriate to that environment, and the cumulative effect of environmental conditions cannot be at once determined. As a result, actual hardware performance data from operating satellites and space hardware is generally considered much more valuable than ground test data, and for most mission-critical hardware, it is preferrable to have been previously flight tested, except in the case of dedicated testing or demonstration missions. We are developing a test bed for the entire closed-loop attitude control system and payload with actual nanosatellite reaction wheel hardware on a 3 -axis rotating air bearing ${ }^{12,13}$ to validate the system as shown in Figs. 6 and 7. To overcome faults and noise injected into the hardware, adaptive terminal sliding mode control laws with a fuzzy system, second order sliding mode control laws with a fuzzy system and third order sliding mode control laws with a fuzzy system are developed for discrete time, and tested on the spherical air bearing system. These controller laws can increase the attitude tracking control accuracy without using redundant reaction wheels. The experimental results show the proposed analytical fault tolerant control laws to be effective.

\section{VI..1. Case 1 Results}

The proposed AFSMC in this paper was applied to an air bearing attitude control testing system. The design parameters of control laws are in Tables 7, and 8. The AFSMC controller was used to perform a 90 degree yaw slew maneuver of a nanosatellite. The only other controller previously tested in this air bearing system was a PID controller. Figs. 8 and 9 show the results of a 90 degree slew of the system. Fig. 10 shows the controller values used. Fig. 11 shows the PWM signal sent to the reaction wheel system during this test. The nanosatellite makes a 90 degree slew with a settling time of roughly 60 seconds, settling on a steady state error of 0.8 degree using AFSMC. The PID controller has a steady state error of approximately 5 degree.

Table 7. PID Controller Parameters for Numerical Analysis

\begin{tabular}{ccc}
\hline \hline Name & Design Parameters & Values \\
\hline Proportional parameters & $k_{p}$ & 0.026 \\
Integral parameters & $k_{i}$ & 0.0001 \\
Derivative parameters & $k_{d}$ & 0.1 \\
\hline \hline
\end{tabular}

Table 8. AFSMC Controller Parameters for Numerical Analysis

\begin{tabular}{l|c}
\hline Parameters & Values \\
\hline$\kappa, \epsilon$ & $0.01,0.0001$ \\
$k_{1}$ & 0.004 \\
$\theta_{0}, \alpha$ & $0,0.1$ \\
\hline
\end{tabular}

Figs. 12 and 13 show the results of a 180 degree slew of the system with actuator fault. The fault occurred between $t=20-120$ s. Two actuator faults were tested. One is the "Reduction Fault" in which output torque is reduced by $50 \%$. Another is the "Constant Fault" in which a constant torque offset of $25 \%$ of maximum is added between $t=20-120 \mathrm{~s}$. Fig. 14 shows the controller values used. Figs. 15 and 16 show the PWM signal sent to the reaction wheel system and the applied motor torque fraction of maximum. The nanosatellite makes a 180 degree slew with a settling time of roughly 150 seconds, settling on a steady state error of 0.8 degree using AFSMC. The PID controller still has a steady state error of approximately 5 degree.

\section{VI..2. Case 2 Results}

In this section, we test the adaptive fuzzy sliding mode controller, PID, 2nd SMC and 3rd SMC with actuator faults. Figs. 17 and 18 show the results of a 90 degree slew of the system. Fig. 19 shows the controller 


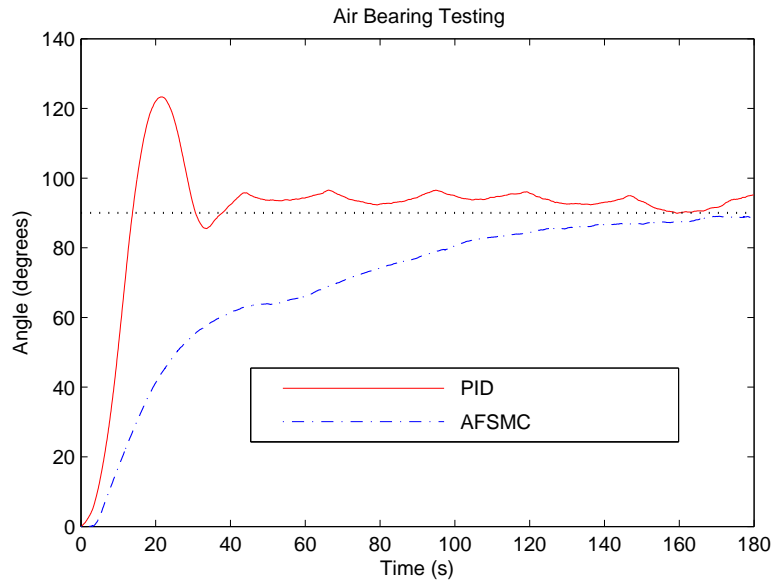

Figure 8. Nanosatellite 90 Degrees Slew

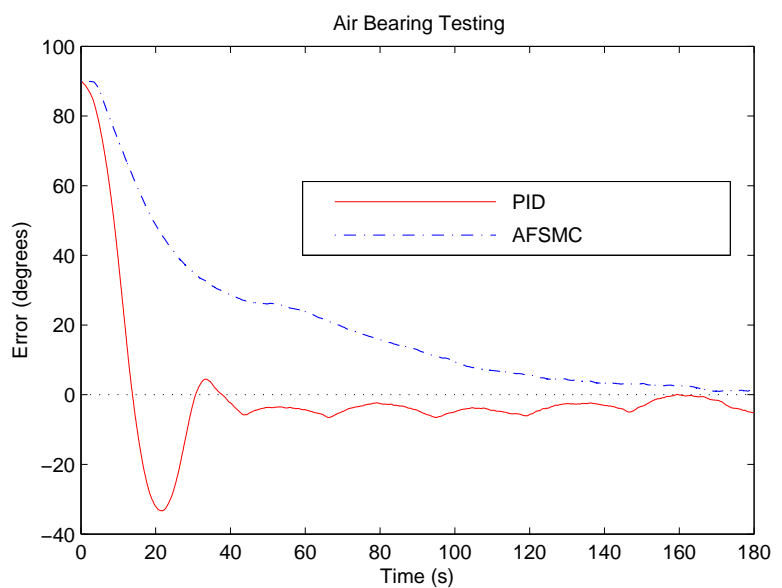

Figure 9. Nanosatellite Tracking Error during 90 Degrees Slew

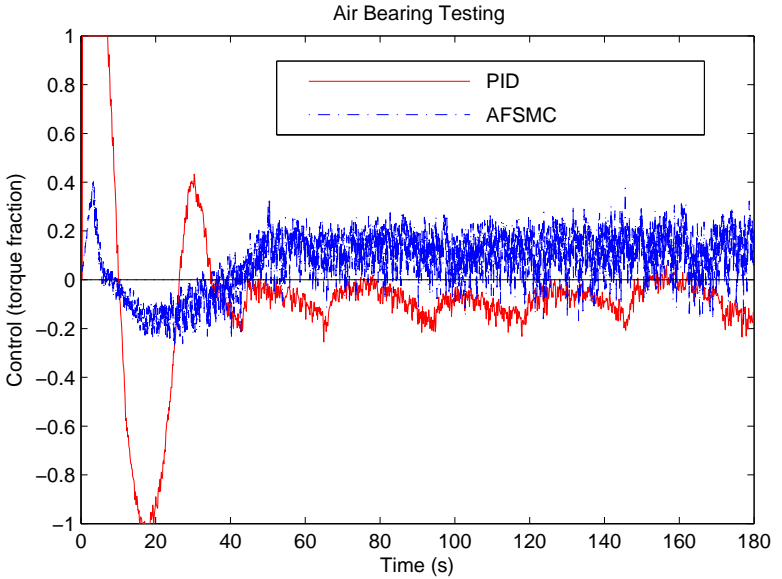

Figure 10. Nanosatellite Control during 90 Degrees Slew 


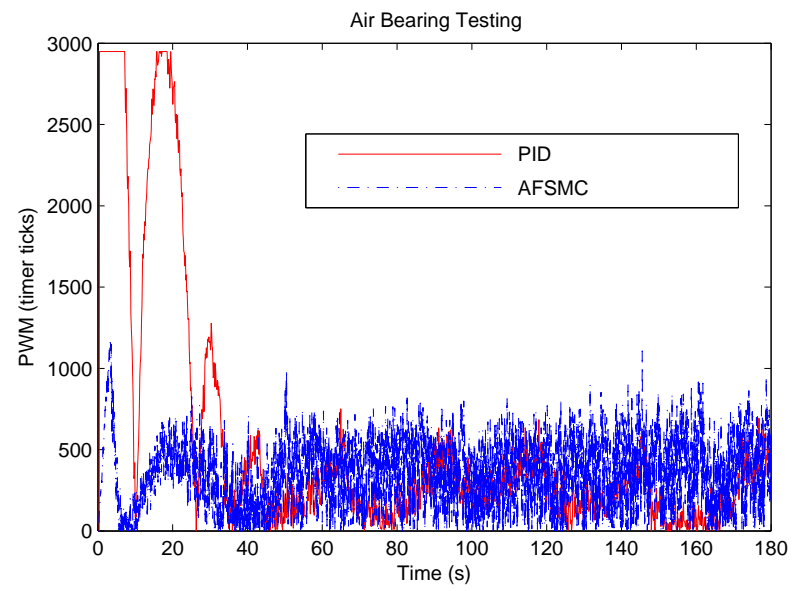

Figure 11. Nanosatellite PWM Signal during 90 Degrees Slew

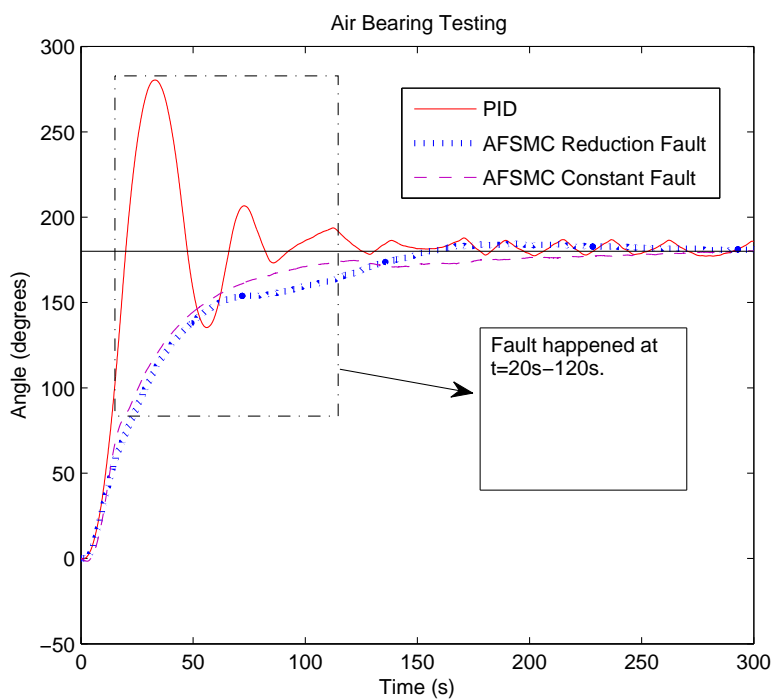

Figure 12. Nanosatellite 180 Degrees Slew with Actuator Faults

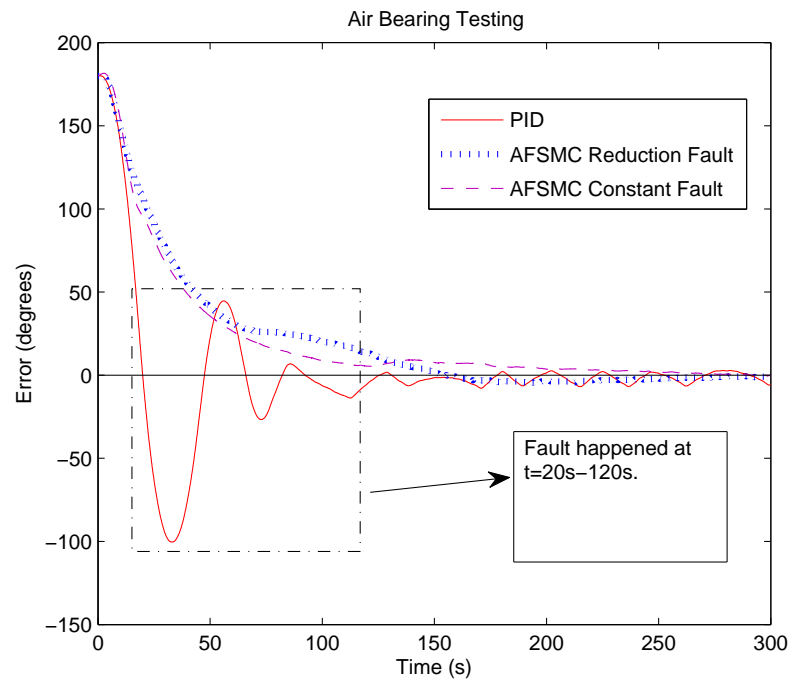

Figure 13. Nanosatellite Tracking Error during 180 Degrees Slew with Actuator Faults 


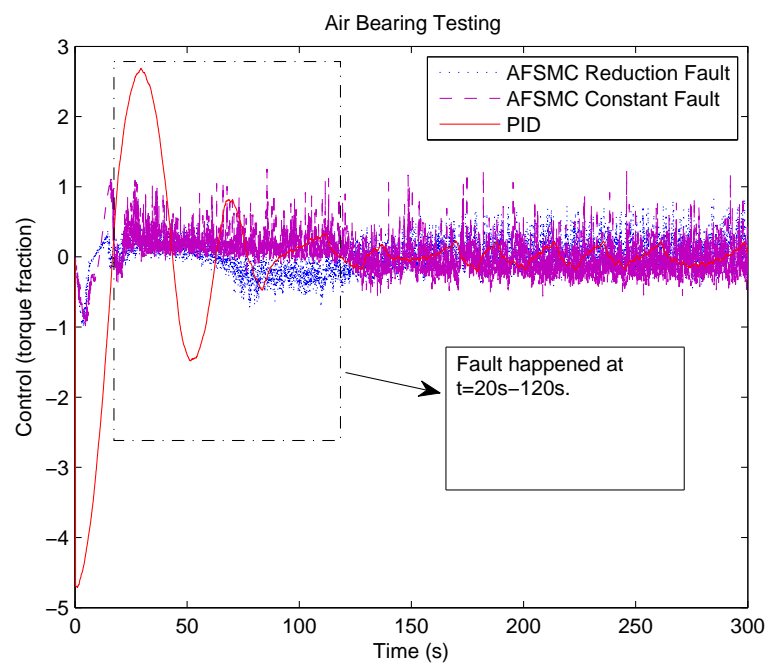

Figure 14. Nanosatellite Control during 180 Degrees Slew with Actuator Faults

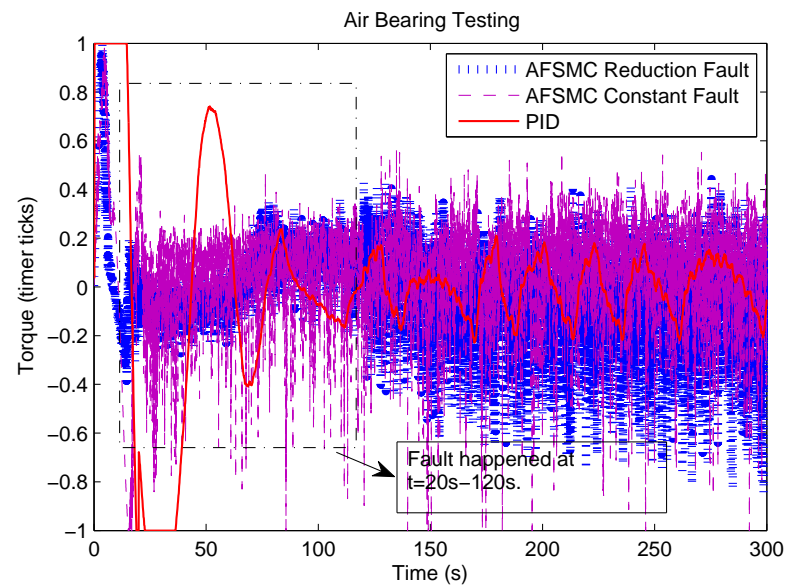

Figure 15. Nanosatellite Torque during 180 Degrees Slew with Actuator Faults

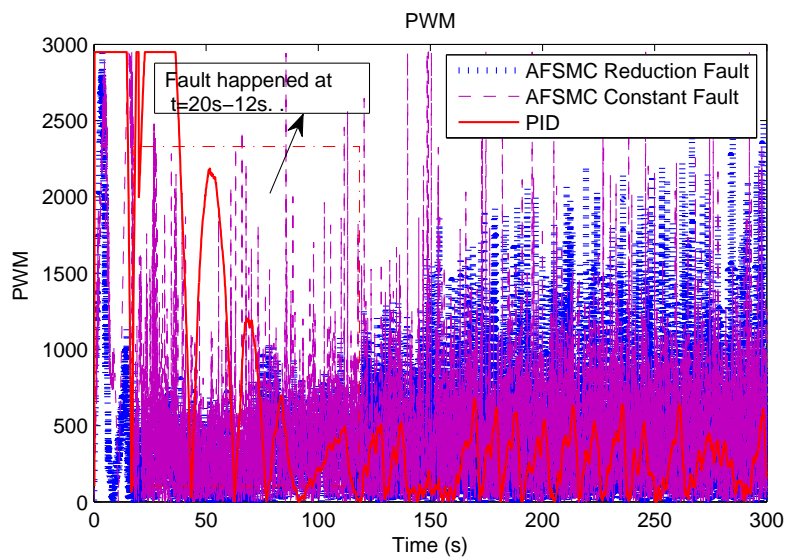

Figure 16. Nanosatellite PWM Signal during 180 Degrees Slew with Actuator Faults 
output during these tests. The tracking errors are nearly the same using all three controllers. The AFSMC controller needs less actuator energy than the other controllers. Fig. 20 shows a longer test for the 90 degree slew maneuver. Next, we added actuator faults using AFSMC, PID, 2nd SMC and 3rd SMC. Figs. 21 and

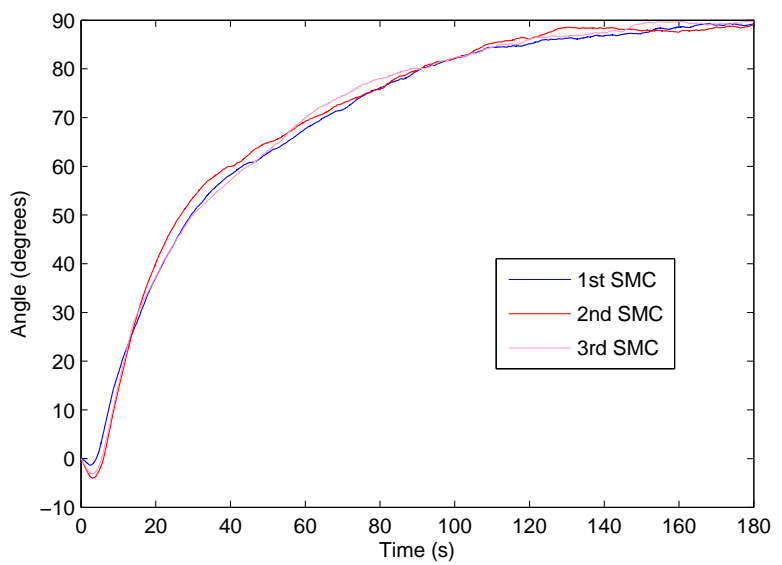

Figure 17. Nanosatellite 90 Degrees Slew with AFSMC, 2nd SMC and 3rd SMC in $180 \mathrm{~s}$

22 show the results and control of a 90 degree slew of the system with the actuator experiencing a "Constant Fault" in which a constant torque offset of $25 \%$ of maximum is added between $t=180-240$ s. Figs. 23 and 24 show the fuzzy logic system and sliding surface values. The dynamic changes due to faults are represented by the fuzzy logic system " $H r=\theta^{T} \xi "$. The sliding motion of " $S_{r p}$ " is broken by the fault and recovers quickly in the controller. The robustness of the proposed AFSMC controller is the best out of the four compared. The PID controller can not handle the faults as effectively as the sliding mode controllers. The 2nd SMC tracking error is not as good as that of AFSMC and 3rdSMC.

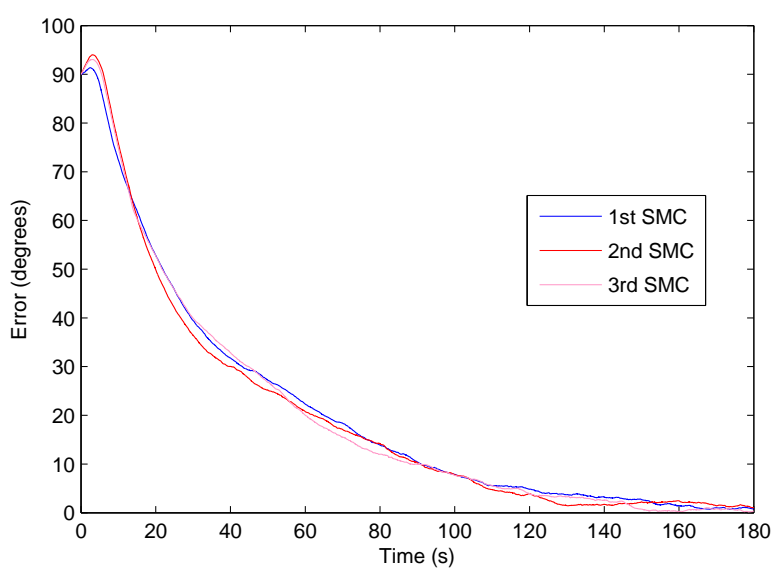

Figure 18. Nanosatellite Tracking Error during 90 Degrees Slew with AFSMC, 2nd SMC and 3rd SMC in 180 s

\section{VI..3. Case 3 Results}

Figs. 25, 26, 27 and 28 show the error and AFSMC/PID control output signal for a 90 degree yaw slew of the system about the z-axis and roll 0 degree. Due to the difficulty of perfectly balancing the air bearing system, a gravitational disturbance, larger than normal in size, is considered to be present about the $\mathrm{x}$ and y rotational axes. The PID controller does not have as good tracking performance as the sliding mode controller. The AFSMC controller uses nearly the same gain and has much better tracking performance.

\section{Conclusions}

Attitude control of small satellites is still predominantly performed by standard controllers such as Proportional-Integral-Derivative controllers. The implementation complexity of nonlinear control hinders 


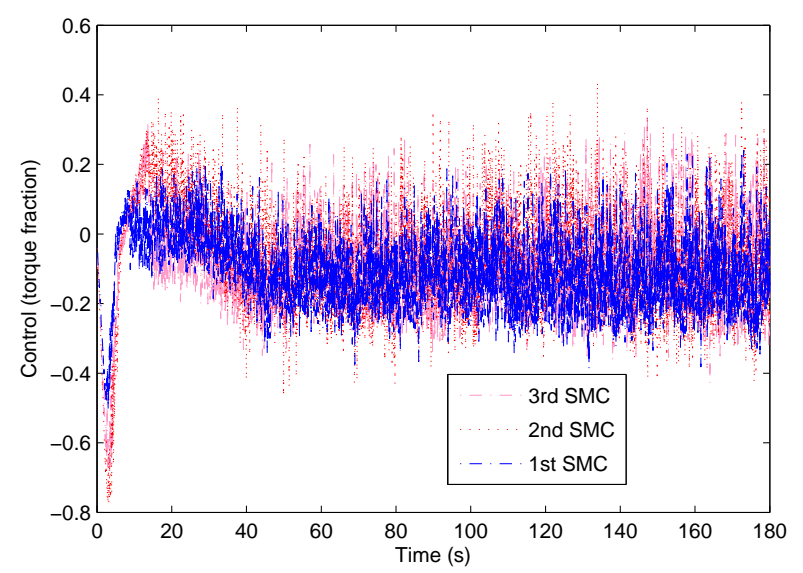

Figure 19. Nanosatellite Control during 90 Degrees Slew with AFSMC, 2nd SMC and 3rd SMC in $180 \mathrm{~s}$

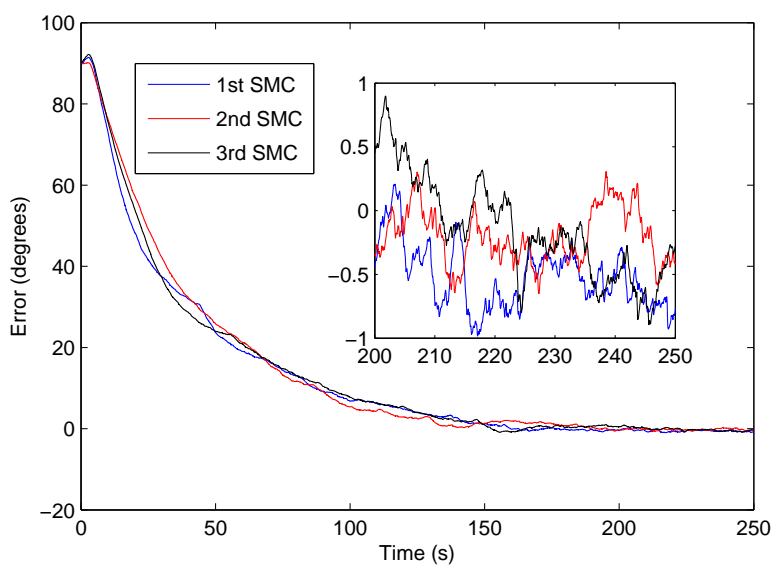

Figure 20. Nanosatellite 90 Degrees Slew with AFSMC, 2nd SMC and 3rd SMC in 250 s

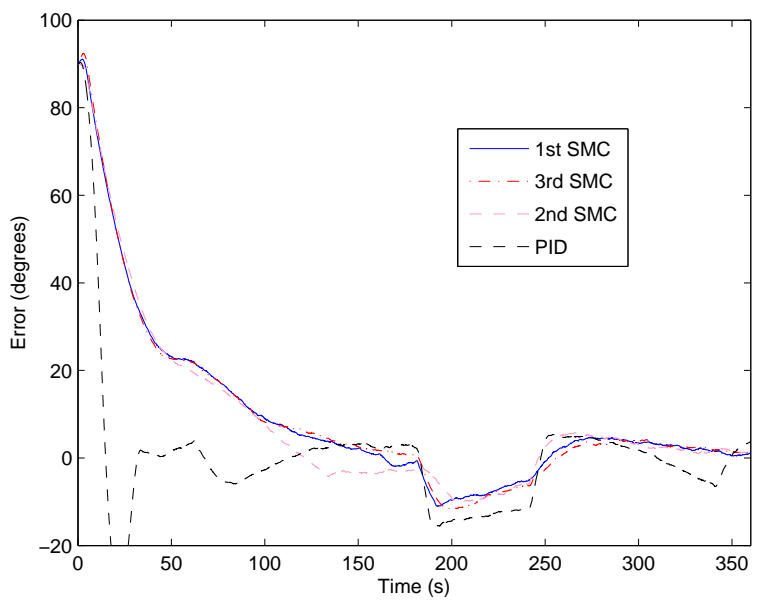

Figure 21. Nanosatellite 90 Degrees Slew tracking errors with AFSMC, 2nd SMC, 3rd SMC and PID in $360 \mathrm{~s}$ 


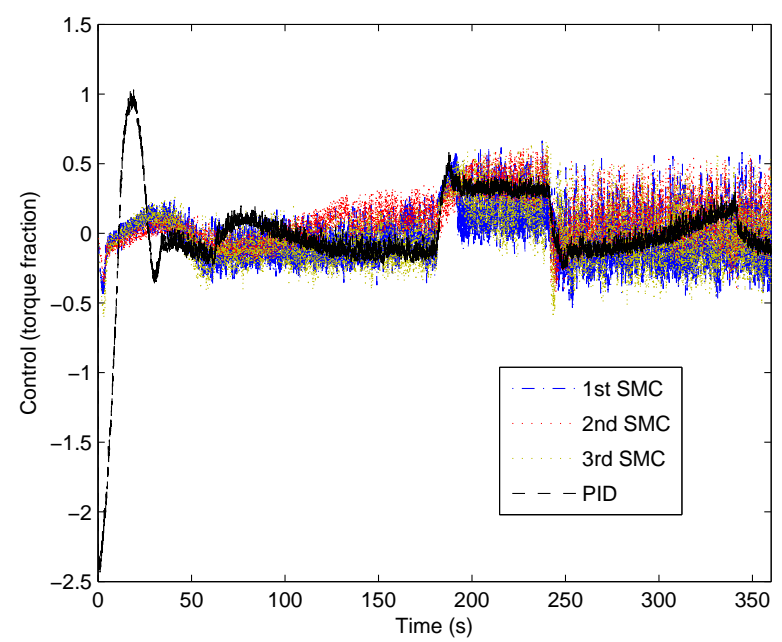

Figure 22. Nanosatellite Control Input using AFSMC, 2nd SMC, 3rd SMC and PID in $360 \mathrm{~s}$

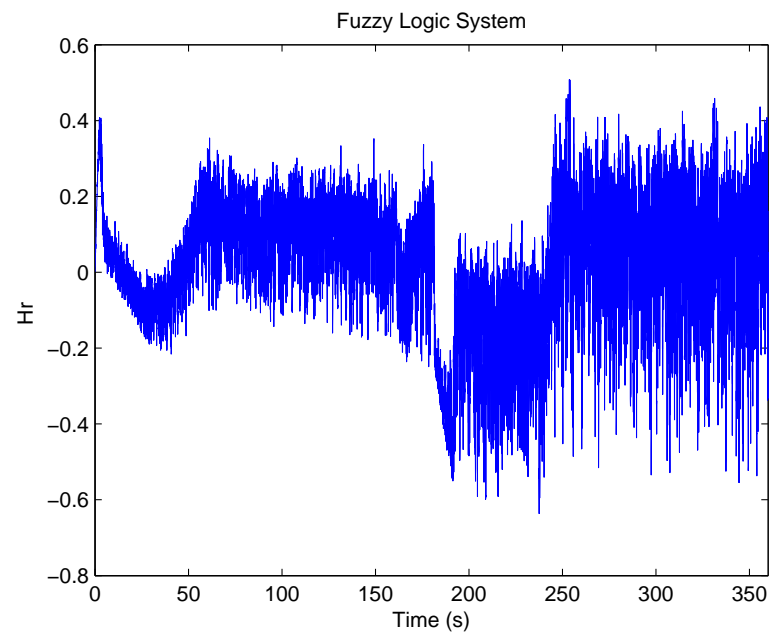

Figure 23. Fuzzy Logic System with AFSMC in $360 \mathrm{~s}$

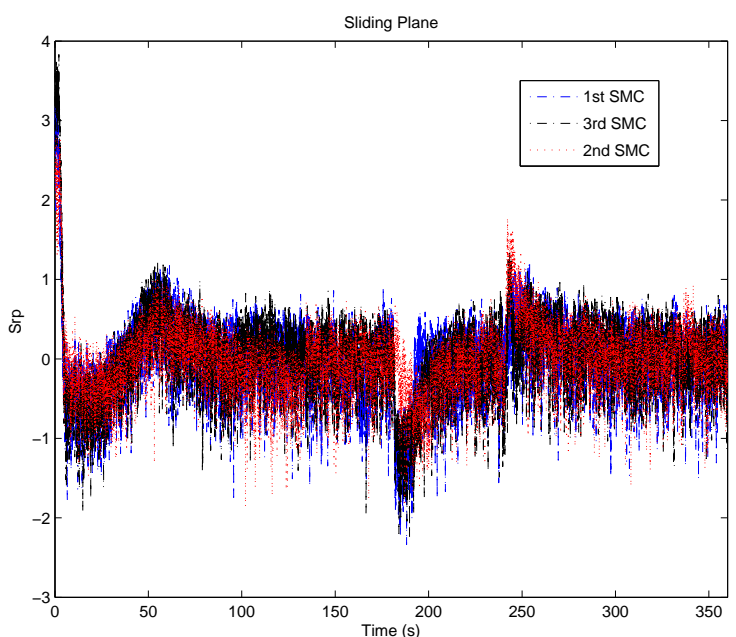

Figure 24. Sliding Motion of AFSMC, 2nd SMC and 3rd SMC in $360 \mathrm{~s}$ 


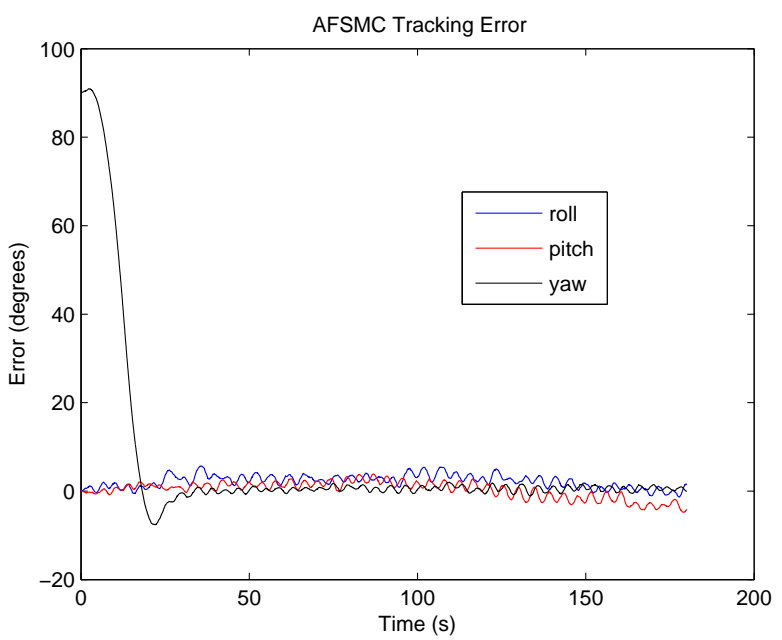

Figure 25. AFSMC Tracking Errors for 3 Axis ACS

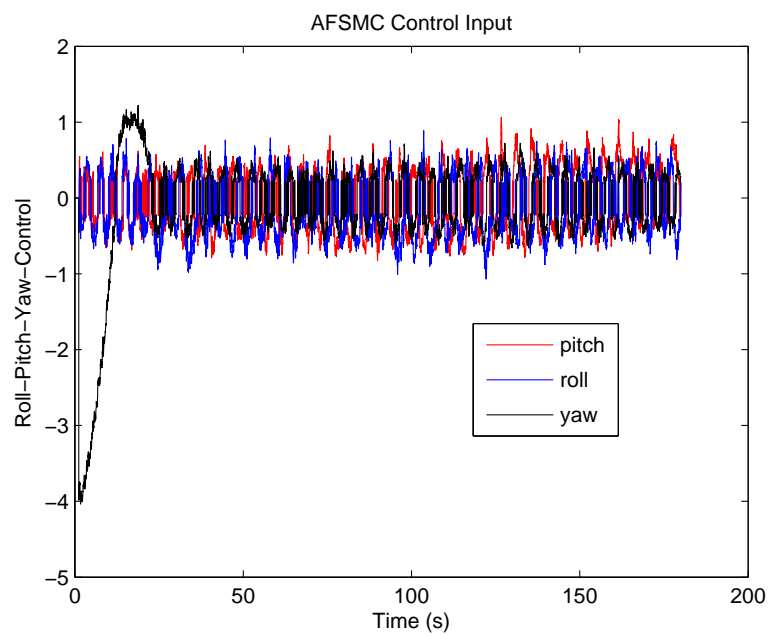

Figure 26. AFSMC control Input for 3 Axis ACS

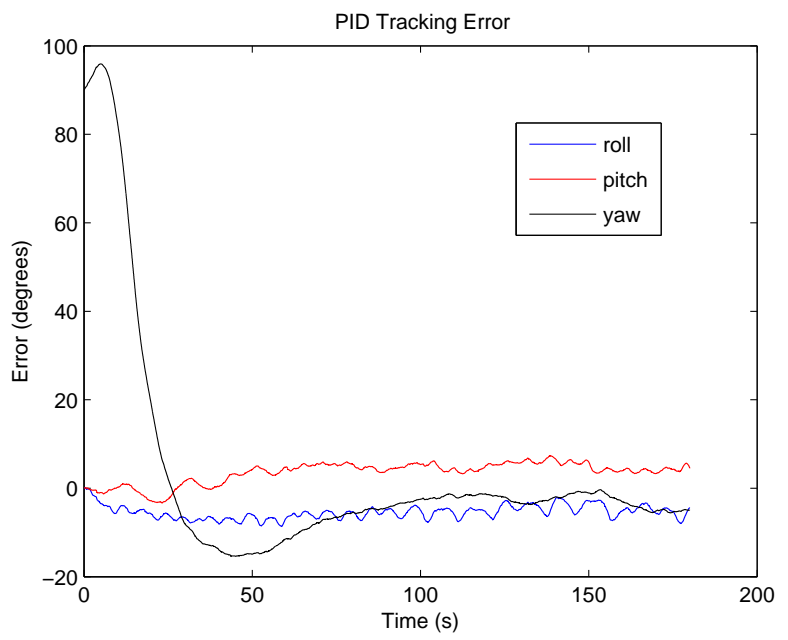

Figure 27. PID Tracking Errors for 3 Axis ACS 


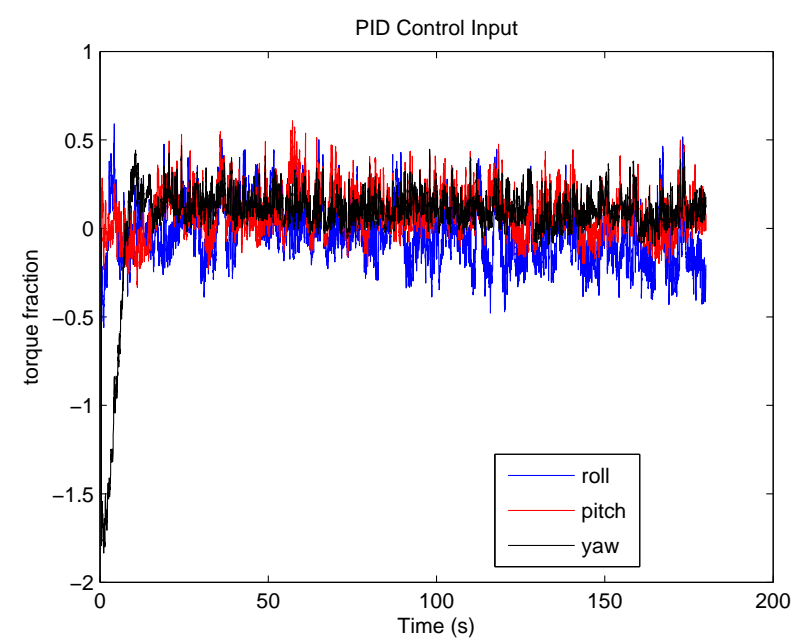

Figure 28. PID control Input for 3 Axis ACS

the development of practical implementations for ACS. In this paper, numerical research on nonlinear control has been performed carefully. The purpose of this paper is to verify the proposed adaptive fuzzy sliding mode control law on an air bearing system. A similar approach with an air bearing system has been used at the Surrey space centre. ${ }^{14}$ However, this work did not include fault tolerant control. The control in ${ }^{14}$ uses PD and optimal control laws. In this paper, nonlinear AFSMC control has been successfully verified on an air bearing simulator. 


\section{References}

${ }^{1}$ Ure, N. K., Kaya, Y. B., and Inalhan, G., "The development of a Software and Hardware-in-the-Loop Test System for ITU-PSAT II nano satellite ADCS," IEEE 2011 Aerospace Conference, Big Sky, MT, 5-12 March 2011.

${ }^{2}$ Won, C.-H., "Comparative Study of Various Control Methods for Attitude Control of a LEO Satellite," Aerospace Science and Technology, , No. 5, 1999, pp. 323-333.

${ }^{3}$ Kim, K.-S. and Kim, Y. D., "Robust Backstepping Control for Slew Maneuver using Nonlinear Tracking Function," IEEE Transactions on Control Systems Technology, Vol. 11, No. 6, 2003, pp. 822-829.

${ }^{4}$ Lo, S. C. and Chen, Y. P., "Smooth Sliding Mode Control for Spacecraft Attitude Tracking Maneuvers," Journal of Guidance Control and Dynamics, Vol. 18, No. 6, 1995, pp. 1345-1349.

${ }^{5}$ Lin, J. Y., Ko, S., and Ryoo, C.-K., "Fault Tolerant Control of Satellites with Four Reaction Wheels," Control Engineering Practice, Vol. 16, 2008, pp. 1250-1258.

${ }^{6}$ Pukdeboon, C., Zinober, A. S. I., and Thein, M. W. L., "Quasi-Continuous Higher Order Sliding mode controllers for spacecraft attitude tracking maneuvers," IEEE Transactions on Industrial Electrionics, Vol. 57, No. 4, 2010, pp. 1436-1444.

${ }^{7}$ Fukuda, K., Nakano, T., Sakamoto, Y., Kuwahara, T., Yoshida, K., and Takahashi, Y., "Fuzzy Bang-bang Relay Controller for Satellite Attitude Control System," Fuzzy Sets and Systems, Vol. 162, 2010, pp. 2104-2125.

${ }^{8} \mathrm{Hou}$, Z.-G., Zou, A.-M., Cheng, L., and Tan, M., "Adaptive Control of an Electrically driven nonholonomic mobile robot via backstepping and fuzzy approach," IEEE Transactions on Control System Technology, Vol. 17, No. 4, 2009, pp. 803-815.

${ }^{9}$ Lee, H. and Kim, Y., "Robust adaptive control using a universal approximator for SISO nonlinear systems," IEEE Transactions on Fuzzy Systems, Vol. 8, No. 1, 2000, pp. 95-106.

${ }^{10} \mathrm{Li}$, J. Q., Post, M. A., and Lee, R., "Nanosatellite Attitude Air Bearing System using Variable Structure Control," IEEE 25th Annual Canadian Conference on Electrical and Computer Engineering, Montreal, Canada, April. 29- May. 2 , 2012.

${ }^{11}$ Levant, A., "Quasi-continuous High Order Sliding Mode Controllers," IEEE Transactions on Automatic Control, Vol. 50, No. 11, 2005, pp. 1812-1816.

${ }^{12}$ Prado, J., Bisiacchi, G., Reyes, L., Vicente, E., Contreras, F., Mesinas, M., and Jurez, A., "Three-Axis Air-Bearing Based Platform for Small Satellite Attitude Determination and Control Simulation," Journal of Applied Research and Technology, Vol. 3, 2005, pp. 222-237.

${ }^{13}$ Kim, J. J. and Agrawal, B. N., "Automatic Mass Balancing of Air-Bearing-Based Three-Axis Rotational Spacecraft Simulator," Journal of Guidance, Control, and Dynamics, Vol. 32, No. 3, 2009, pp. 1005-1017.

${ }^{14}$ Horri, N. M., Plamer, P. L., and Robers, M., "Energy Optimal Spacecraft Attitude Control Subject to Convergence rate Constraints," Control Engineering Practice, Vol. 19, 2011, pp. 1297-1314. 